\title{
Solution of the Problem of Flow Past a Wing Profile Near the Interface
}

\author{
Ludmila V. Kulagina ${ }^{a}$, \\ Vladimir A. Kulagin ${ }^{a}$ and Feng-Chen $\mathbf{L i}^{b^{*}}$ \\ ${ }^{a}$ Siberian Federal University \\ 79 Svobodny, Krasnoyarsk, 660041, Russia \\ ${ }^{b}$ School of Energy Science and Engineering \\ Harbin Institute of Technology \\ Harbin 150001, China
}

Received 26.02.2017, received in revised form 14.04.2017, accepted 26.05.2017

In this paper presente some approaches to solving the problems of flow past a wing profile near the interface. Studies on the flow in the vicinity of separation boundaries (solid walls and free surfaces) show that in a confined flow liquid turns into a bubble mixture of liquid and gas. This complicates the flow analysis and introduces additional losses resulting in impaired energy performance of the concerned mechanisms. In a general case the problem of a two-phase compressible flow around various types of vane mechanisms is substantially nonlinear (even under no-vortex flow assumption).

Keywords: flow around actual foil-shaped profiles, supercavitating mechanisms near separation boundary, potential theory, method solution.

Citation: Kulagina L.V., Kulagin V.A., Li F.-Ch. Solution of the problem of flow past a wing profile near the interface, J. Sib. Fed. Univ. Eng. technol., 2017, 10(4), 523-533. DOI: 10.17516/1999-494X-2017-10-4-523-533.

(c) Siberian Federal University. All rights reserved

* Corresponding author E-mail address: klvation@gmail.com, v.a.kulagin@mail.ru, lifch@hit.edu.cn 


\title{
Решение задачи обтекания крылового профиля вблизи границы раздела
}

\author{
Л.В. Кулагина ${ }^{a}$, В.А. Кулагин ${ }^{a}$, Ф.-Ч. Ли \\ ${ }^{a}$ Сибирский федеральный университет \\ Россия, 660041, Красноярск, пр. Свободный, 79 \\ ${ }^{\sigma}$ Школа энергетических наук и техники \\ Харбинский технологический институт \\ Харбин 150001, Китай
}

В настоящей работе представлены некоторые подходы к решению задач обтекания крылового профиля вблизи границыь раздела. Исследования потока вблизи границ раздела (сплошные стенки и свободные поверхности) показывают, что в замкнутом потоке жидкость превращается в пузырьковую смесь жидкости и газа. Это усложняет анализ потока и вносит дополнительные потери, приводящче к ухудшению энергетических характеристик соответствующих механизмов. В общем случае проблема двухфазного сжимаемого течения вокруг различных типов лопастных механизмов существенно нелинейна (даже при предположении отсутствия вихревого потока).

Ключевые слова: обтекание крылового профиля, суперкавитационные механизмы, границы раздела, теория потенциала, метод решения.

\section{Introduction}

Most of the problems in potential theory [1] can generally be reduced to solving integral or integral differential equations or sets of such equations.

In the case of small perturbations, the solution to the problem comes down to solving the integral equation $[3,4]$

$$
\frac{1}{2 \pi} \int_{=1}^{+1} \gamma(s)\left\{\frac{1}{\xi-s}-G_{1}\right\} \mathrm{d} s=-F_{\mathrm{cp}}(\xi)-\frac{1}{2 \pi} \int_{-1}^{+1} \chi(s) G_{2} \mathrm{~d} s,
$$

where $\chi$ and $F_{\mathrm{cp}}$ are functions of the hydrofoil shape and kernels $D_{1}$ and $G_{2}$ are given by $G_{1}=(\xi-s) / \Delta ; G_{2}=\varepsilon / \Delta ; \Delta(\xi-s)^{2}+\varepsilon^{2} ; \quad \varepsilon=4 \bar{h}(0)$. The foil shape is defined as $F=F_{\mathrm{H}} \pm F_{\mathrm{c}}$, where $F_{\mathrm{H}}$ is the centerline equation and $F_{\mathrm{c}}$ is the thickness distribution. In the first approximation of the no-penetration boundary condition we obtain:

a) $F_{\mathrm{cp}}^{(1)}(\xi)=-\alpha+\sum_{0}^{N} a_{n} \xi^{n}$, if $F_{H}=\sum_{0}^{N} \frac{a_{n}}{n+1} \xi^{n+1}$

b) $\chi^{(1)}(\xi)=2 w(\xi) \sum_{0}^{M} b_{n} \xi^{n}+2 w^{\prime}(\xi) \sum_{0}^{M} \frac{b_{n}}{n+1} \xi^{n+1} \quad$ if $\quad F_{\mathrm{c}}=\sum_{0}^{M} \frac{b_{n}}{n+1} \xi^{n+1} w(\xi)$, where $w=1$ for a sharp leading edge and $w=\sqrt{1-\xi^{2}}$ for a rounded one.

Equation (1) is an integral Fredholm equation of the first kind with a singularity in its kernel

$$
\int_{a}^{b} k(\xi, s, \varepsilon) \gamma(s) \mathrm{d} s=f(\xi) .
$$


The solution $\gamma$ of Eq. (2) is known [6] to be unstable even at small errors in $f(\xi)$ data and sensitive to errors in the kernel $k(\xi, s)$ and it is virtually independent of the solving technique.

The problem itself, (1), is essentially ill-defined and requires proper regularization techniques to enable its solution [6]. What makes it ill-posed when $\bar{h} \rightarrow 0$ is that integral equation (1) degenerates to a differential equation that is linear with respect to the higher derivative. By means of unsophisticated mathematics [3, 4] integral equation (1) of the flow boundary-value problem is rewritten as

$$
\frac{\varepsilon^{2}}{2 \pi} \sum_{n=1}^{n} \frac{(-1)^{n}}{n !} \gamma^{(n)}(\xi) W_{\alpha}^{n}(\xi, \varepsilon)+\frac{1}{2 \pi} \gamma(\xi) W_{1}\langle\xi, s\rangle=\Phi(\xi),
$$

where $\Phi(\xi)=-F_{\mathrm{cp}}(\xi)-\frac{1}{2 \pi} \chi(\xi) W_{0}(\xi, \varepsilon)-\frac{1}{2 \pi} \sum_{n+1}^{N} \frac{(-1)^{n}}{n !} \chi_{(\xi)}^{(n)} W_{\delta}^{n}(\xi, \varepsilon)$

$W_{\alpha}^{n}(\xi, \varepsilon)=\int_{-1+\xi}^{1+\xi} \frac{U^{n-1} \mathrm{~d} U}{U^{2}+\varepsilon^{2}}$

$W_{1}(\xi, \varepsilon)=\int_{-1}^{+1}\left\{\frac{1}{\xi-s}-G_{1}\right\} \mathrm{d} s=\ln \frac{1+\xi}{1-\xi} \sqrt{\frac{(1-\xi)^{2}+\varepsilon^{2}}{(1+\xi)^{2}+\varepsilon^{2}}} ;$

$W_{0}(\xi, \varepsilon)=\int_{-1}^{+1} G_{2} \mathrm{~d} s=\operatorname{arctg} \frac{1-\xi}{\varepsilon}+\operatorname{arctg} \frac{1+\xi}{\varepsilon} ;$

$W_{\delta}^{n}(\xi, \varepsilon)=\int_{-1}^{+1}(\xi-s)^{n} G_{2} \mathrm{~d} s, \quad \varepsilon=4 \bar{h}(0)$.

From Eq. (3) it follows that when $\bar{h} \rightarrow 0$, problem (1) reduces to a boundary-layer-type problem, which sheds light on why the problem is ill-posed. The physical conditions impose limitation on $N$.

This problem can be solved employing a hybrid approach. First, solutions to the exterior, (1), and interior, (3), problems are found [2,3] and then these are mutually adjusted.

There is yet another way to tackle the problem. It starts with constructing a perturbed exterior solution and then this solution, which is ill-suited for $\bar{h} \rightarrow 0$, is transformed so that it is able to reveal the nature of singularity. The resultant solution thus becomes uniformly valid everywhere and provides good approximation to the true solution. The solution can be further improved quantitatively via higher-order approximations and, finally, nonlinear methods can be applied to accelerate convergence of the functional sequence $[3,4]$.

If the sequence is divergent, which depends on the class of the function $f(\xi)$ in $(1)$, then the linear transformations and algorithms discussed above allow the main term of the sequence to be extracted. Write the solution of integral equation (1) as $\gamma=\gamma_{1}+\gamma_{2}$. The first term is associated with the influence of the centerline shape on circulation, while the second one is attributed to the dynamic curvature resulting from the flow around a profile near the separation boundary.

\section{The functional parameter method}

Represent $\gamma_{1}$ solution in the form of a $\tau=\sqrt{1+4 \bar{h}^{2}}-2 \bar{h}$ series obtained by mapping $\bar{h} \in[0, \infty]$ to $\tau \in[1,0]$ : 


$$
\gamma_{1}^{(n)}=\sum_{m=0}^{N} \gamma_{1(2 m)}^{(n)} \tau^{2 m}
$$

There is an expansion for the kernel with respect to parameter $\tau$ such that:

$$
G_{1}=\sum_{m=1}^{N} k_{1 m} \tau^{2 m}
$$

with $k_{1 m}$ defined in [3].

Series (5) is convergent. Moreover, it is convergent over the entire actual range of variation of the parameter $r$. Convergence of series (4) remains questionable because it is not possible to construct a general term. It is however possible to evaluate a convergence domain for specific foil shapes.

Substituting (4) and (5) into (1) and resolving the solution into two terms (terms of the same $\tau$ power are taken equal) yields a system of singular integral equations with a Cauchy-type kernel:

$$
\int_{-1}^{+1} \gamma_{1(2 m)}^{(n)}(s) \frac{\mathrm{d} s}{\xi-s}=\Phi_{m}^{(n)}(\xi), m=0,1,2, \ldots N ; \quad n=1,2, \ldots
$$

Converting this equation into $a_{\infty}^{0}$ class functions we have

$$
\gamma_{1(2 m)}^{(n)}(\xi)=-\frac{1}{\pi^{2}} \sqrt{\frac{1-\xi}{1+\xi}} \int_{-1}^{+1} \sqrt{\frac{1-\xi}{1+\xi}} \frac{\Phi_{m}^{(n)}(s)}{\xi-S} \mathrm{~d} s .
$$

The function $\Phi_{m}^{(n)}(m=1,2, \ldots, N)$ is found from the boundary conditions via the solutions $\gamma_{1(2 m)}^{(n)}(m=1,2, \ldots, N-1)$. Let us now write the solutions for $N=13$ and $n=1$ for a plate when $F_{\mathrm{cp}}^{(1)}=-\alpha$

$$
\begin{aligned}
& \Phi_{0}^{(1)}=-2 \pi \alpha ; \quad \Phi_{1}^{(1)}=\int_{-1}^{+1} \gamma_{10}^{(1)} k_{11} \mathrm{~d} s ; \\
& \Phi_{2}^{(1)}=\int_{-1}^{+1}\left\{\gamma_{10}^{(1)} k_{12}+\gamma_{12}^{(1)} k_{11}\right\} \mathrm{d} s ; \\
& \Phi_{m}^{(1)}=\int_{-1}^{+1}\left\{\sum_{1}^{m} \gamma_{1[2(k-1)}^{(1)} k_{1(m-k+1)}\right\} \mathrm{d} s .
\end{aligned}
$$

Substituting (8) into (7) gives

$$
\gamma_{10}^{(1)}(\xi)=2 \alpha \sqrt{\frac{1-\xi}{1+\xi}} ; \quad \gamma_{1(2 m)}^{(1)}=\gamma_{10}^{(1)} W_{2 m}
$$

where $m=1,2, \ldots, 13$;

$W_{2}=\frac{3}{2}+\xi$;

$W_{4}=\frac{9}{8}-\frac{1}{2} \xi-\frac{5}{2} \xi^{2}-\xi^{3}$;

$W_{6}=\frac{15}{16}-\frac{9}{8} \xi-\frac{5}{4} \xi^{2}+3 \xi^{3}+\frac{7}{2} \xi^{4}+\xi^{5}$;

$W_{8}=\frac{105}{128}-\frac{1}{16} \xi+\frac{53}{16} \xi^{2}+\frac{43}{8} \xi^{3}-\frac{21}{8} \xi^{4}-\frac{15}{2} \xi^{5}-\frac{9}{2} \xi^{6}-\xi^{7}$; 


$$
\begin{aligned}
& W_{10}=\frac{255}{256}+\frac{217}{128} \xi+\frac{145}{32} \xi^{2}-\frac{19}{4} \xi^{3}-\frac{165}{8} \xi^{4}-\frac{33}{4} \xi^{5}+\frac{27}{2} \xi^{6}+14 \xi^{7}+\frac{11}{2} \xi^{8}+\xi^{9} \\
& W_{12}=\frac{1227}{1024}+\frac{471}{256} \xi-\frac{617}{256} \xi^{2}-\frac{2553}{128} \xi^{3}-\frac{889}{64} \xi^{4}+\frac{305}{8} \xi^{5}+\frac{415}{8} \xi^{6}-\frac{3}{4} \xi^{7}-\frac{275}{8} \xi^{8}- \\
& -\frac{45}{2} \xi^{9}-\frac{13}{2} \gamma^{10}-\xi^{11} \\
& W_{14}=\frac{2613}{2048}-\frac{263}{1024} \xi-\frac{5825}{512} \xi^{2}-\frac{1631}{128} \xi^{3}+\frac{11943}{256} \xi^{4}+\frac{10773}{128} \xi^{5}-\frac{837}{32} \xi^{6}-\frac{553}{4} \xi^{7}- \\
& -\frac{1295}{16} \xi^{8}+\frac{305}{8} \xi^{9}+\frac{273}{4} \xi^{10}+33 \xi^{11}+\frac{15}{2} \xi^{12}+\xi^{13} \\
& W_{16}=\frac{36297}{32768}-\frac{5617}{2048} \xi-\frac{20883}{2048} \xi^{2}+\frac{27063}{1024} \xi^{3}+\frac{99597}{1024} \xi^{4}-\frac{6939}{256} \xi^{5}-\frac{73617}{256} \xi^{6}- \\
& -\frac{20669}{128} \xi^{7}++\frac{32835}{128} \xi^{8}+\frac{5325}{16} \xi^{9}+\frac{1035}{16} \xi^{10}-\frac{101}{8} \xi^{11}-\frac{945}{8} \xi^{12}-\frac{91}{2} \xi^{13}-\frac{17}{2} \xi^{14}-\xi^{15} \text {; } \\
& W_{18}=\frac{4500439}{65536}+\frac{2374493}{32768} \xi+\frac{81825}{1024} \xi^{2}+\frac{295437}{2048} \xi^{3}+\frac{13037}{128} \xi^{4}-\frac{100183}{512} \xi^{5}-\frac{25677}{128} \xi^{6}+ \\
& +\frac{165239}{256} \xi^{7}+\frac{33753}{32} \xi^{8}+\frac{2049}{8} \xi^{9}-\frac{20049}{32} \xi^{10}-594 \xi^{11}+77 \xi^{12}+294 \xi^{13}+187 \xi^{14}+ \\
& +60 \xi^{15}+\frac{19}{2} \xi^{16}+\xi^{17} \\
& W_{20}=-\frac{12616275}{262144}-\frac{5179823}{65536} \xi-\frac{8336061}{65536} \xi^{2}-\frac{5550025}{32768} \xi^{3}-\frac{3159457}{8192} \xi^{4}-\frac{745381}{1024} \xi^{5}+ \\
& +\frac{149741}{1024} \xi^{13}-\frac{1885}{4} \xi^{14}-\frac{1151}{2} \xi^{15}-\frac{2223}{8} \xi^{16}-\frac{153}{2} \xi^{17}-\frac{21}{2} \xi^{18}-\xi^{19} \text {; } \\
& W_{22}=\frac{132266203353}{131072}+\frac{317275015003}{262144} \xi+\frac{158781624145}{131072} \xi^{2}+\frac{28124698217}{16384} \xi^{3}+ \\
& +\frac{112600162755}{65536} \xi^{4}+\frac{67212868461}{32768} \xi^{5}+\frac{420259071}{2048} \xi^{6}+\frac{2705060269}{2048} \xi^{7}+\frac{2688570425}{2048} \xi^{8}+ \\
& +\frac{80850475}{265} \xi^{9}+\frac{41139813}{128} \xi^{10}+\frac{6691095}{256} \xi^{11}+\frac{5094639}{256} \xi^{12}-\frac{299933}{64} \xi^{13}-\frac{66759}{16} \xi^{14}- \\
& -\frac{1085}{2} \xi^{15}+\frac{20835}{16} \xi^{16}+\frac{8091}{8} \xi^{17}+\frac{1575}{4} \xi^{18}+95 \xi^{19}+\frac{23}{2} \xi^{20}+\xi^{21} \\
& W_{24}=\frac{17129979402909}{4194304}+\frac{1241572992573}{262144} \xi+\frac{1889838437643}{524288} \xi^{2}+\frac{1355616985531}{262144} \xi^{3}+ \\
& +\frac{1311925791219}{262144} \xi^{4}+\frac{396503371015}{65536} \xi^{5}+\frac{388978944203}{65536} \xi^{6}+\frac{106117200107}{32768} \xi^{7}+ \\
& +\frac{58073081641}{16384} \xi^{8}+\frac{116789059}{512} \xi^{9}+\frac{337015355}{512} \xi^{10}-\frac{94300235}{512} \xi^{11}-\frac{28915279}{512} \xi^{12}- \\
& -\frac{4595813}{64} \xi^{13}-\frac{612715}{16} \xi^{14}-\frac{1086565}{16} \xi^{15}-\frac{9434469}{128} \xi^{16}-\frac{13413}{16} \xi^{17}-\frac{45135}{16} \xi^{18}- \\
& -\frac{13185}{8} \xi^{19}-\frac{4301}{8} \xi^{20}-\frac{231}{2} \xi^{21}-\frac{25}{2} \xi^{22}-\xi^{23}
\end{aligned}
$$

If we do not go beyond linearized formulation of the problem, then with (4) and (9) we can find the resultant aerodynamic characteristics - the lift force and pitching momentum coefficients - using the following formulas

$$
C_{Y}^{(n)}=\int_{-1}^{+1} \gamma^{(n)} \mathrm{d} s ; \quad C_{\mathrm{M}}^{(n)}=\int_{-1}^{+1} \gamma^{(n)}(s) s \mathrm{~d} s .
$$


In the first boundary conditions approximation, at $n=1$ we get the functions of influence of the distance parameter $\bar{h}(0)$ on $C_{Y}^{(1)}$ and $C_{\mathrm{M}}^{(1)}$ in the form of $\tau$-series:

$$
\begin{aligned}
& \psi_{Y}^{(1)}=\frac{C_{\gamma}^{(1)}}{C_{\gamma \infty}^{(1)}}=1+\varepsilon+\frac{1}{2} \varepsilon^{2}+\frac{3}{4} \varepsilon^{3}+\frac{23}{32} \varepsilon^{4}+\frac{11}{16} \varepsilon^{5}+\frac{39}{64} \varepsilon^{6}+\frac{163}{256} \varepsilon^{7}+\frac{5491}{8192} \varepsilon^{8}+ \\
& +\frac{2244877}{32768} \varepsilon^{9}-\frac{5379225}{65536} \varepsilon^{10}+\frac{528804484515}{524288} \varepsilon^{11}+\frac{7641989002175}{2097152} \varepsilon^{12}+ \\
& +\frac{37205275391907}{16777216} \varepsilon^{13}+\ldots ; \\
& \psi_{\mathrm{M}}^{(1)}=\frac{C_{\mathrm{M}}^{(1)}}{C_{\mathrm{M} \infty}^{(1)}}=1+\frac{1}{2} \varepsilon+\frac{1}{2} \varepsilon^{2}+\frac{7}{16} \varepsilon^{3}+\frac{15}{32} \varepsilon^{4}+\frac{15}{32} \varepsilon^{5}+\frac{29}{64} \varepsilon^{6}+\frac{899}{2048} \varepsilon^{7}+ \\
& +\frac{3587}{8192} \varepsilon^{8}+\frac{192315}{16384} \varepsilon^{9}-\frac{1604301}{6536} \varepsilon^{10}+\frac{128261286621}{524288} \varepsilon^{11}+ \\
& +\frac{2091064690271}{2097152} \varepsilon^{12}+\frac{4259769570263}{8388608} \varepsilon^{13}+\ldots,
\end{aligned}
$$

where $\varepsilon=\tau^{2}, C_{\gamma \infty}^{(1)}=2 \pi \alpha, C_{\mathrm{M} \infty}^{(1)}=-\pi \alpha$.

Under formal application of the discussed method, the solution $\gamma$ given by (4)(9) is unstable [6]. It is considered that a coarse solution can be obtained taking just a few terms of the series; a further increase in the number of terms will only enhance the instability and the resultant multi-term series will have nothing to do with the true solution of Eq. (1). If however this instability is treated as a transient process, then it can be asserted that increasing the number of approximation terms provides additional information on the behavior of the true solution.

Let $\psi(\varepsilon)$ be an analytical function, then expression (11) can be treated as a Taylor-series expansion. The asymptotic behavior of coefficients of this expansion $\psi=\Sigma a_{n} \varepsilon^{n}$ is determined by the type of the function singularity. So it is natural to look for a way to deduce singularity parameters from a limited sequence $\left\{a_{n}\right\}$ of coefficients of the series [7].

The radius of convergence of the Taylor series is determined by the singularity of the function $\psi(\varepsilon)$ that is closest to the point around which expansion is performed. Farther away singularities may appear to influence the behavior of the series coefficients as well. If this is the case, one should find the spectrum of singularities.

The algorithms considered above offer a solution to the problem of $\psi(\varepsilon)$ synthesis.

Numerical solution of Eq. (1).

Integral equation (1) can be solved by reducing it to a set of algebraic equations (discrete singularities method, collocation method and others). The mentioned methods are strongly unstable [6] as they use conventional quadrature formulas. Attempts to improve the result by increasing the number of nodes when $\varepsilon \rightarrow 0$ only aggravate the situation.

Solution of singularity equation (4) relies on regularization techniques, hence a proper choice of regularizator can essentially enhance stability of the computation scheme.

The structure of the solution $\gamma(\xi)=\sqrt{(1-\xi) /(1+\xi)} \cdot v(\xi)$, where $v(\xi)$ is a non-zero regular function for $\xi= \pm 1$, is determined by a specific behavior of the edge flow. After substitution of this solution into (1) and some simple manipulations we have 


$$
+\frac{\varepsilon^{2}}{2 \pi} \int_{-1}^{+1} \sqrt{\frac{1-s}{1+s}} \frac{\nu(s) \psi(\xi, s) \mathrm{d} s}{\xi-s}=\Phi(\xi),
$$

where $\psi(\xi, s)=1 / \Delta$ and $\Phi(\xi)$ is the right-hand side of Eq. (1).

With the nodes, reference points and quadrature coefficients chosen by the formulas

$$
\begin{aligned}
& \xi_{i}=\cos \theta_{i} ; s_{j}=\cos \theta_{j} ; \quad \theta_{i}=\pi(2 i-1) /(2 N+1), \quad(i=1,2, \ldots, N) ; \theta_{j}=2 \pi j /(2 N+1) ; \\
& A_{j}=2 \pi \sqrt{1-s_{j}^{2}} /(2 N+1),(j=1,2, \ldots, N),
\end{aligned}
$$

we get the following set of algebraic equations

$$
\sum_{j=1}^{N} A_{i, j} Y_{j}=b_{i}, \quad(i, j=1,2, \ldots, N \text { is the number of nodes })
$$

where

$$
\begin{aligned}
& A_{i j}=\psi\left(\xi_{i}, s_{i}, \varepsilon\right) /\left(\xi_{i}-s_{j}\right) ; \quad Y_{j}=\left(1-s_{j}\right) v\left(s_{j}\right) /(2 N+1) ; \\
& b_{i}=-F_{c p}\left(\xi_{i}\right) / \varepsilon^{2}-\sum_{J=1}^{N} \chi\left(s_{i}\right) \sqrt{1-s_{j}^{2}} \psi\left(\xi_{i}, s_{j}, \varepsilon\right) / \varepsilon(2 N+1) ; \psi=1 / \Delta ; \Delta=\left(\xi_{\mathrm{i}}-s_{j}\right)^{2}+\varepsilon^{2}
\end{aligned}
$$

Aerodynamic characteristics of a foil are derived from formulas (10). Switching to the quadrature formulas gives

$$
C_{Y} \cong 2 \pi \sum_{j=1}^{N} Y_{j} \text { and } C_{M} \cong 2 \pi \sum_{j=1}^{N} Y_{j} s_{j}
$$

The BCF algorithm is a powerful tool to handle integrals and resolve sets of algebraic equations due to the quadrature and cubature formulas

$$
S=T\left(\sum_{k=1}^{n} \bar{P}_{k} S\left(\bar{x}_{k}\right)\right)+R,
$$

( $\bar{P}_{k}$ - weights, $\bar{x}_{k}$ - nodes, $\mathrm{R}$ - remainder) and the Nedashkovsky-Skorobogatko BCF method [8] acting as regularizators in the process. The advantage of this technique $[8,9]$ is that it is inherently selfregularized because of mutual cancellation of computational errors and as such it is little sensitive to adverse changes in the coefficient matrix conditioning. A good result is obtained when the BCF method is employed to accelerate convergence or find an antilimit of sequences. To that end, a set of equations is constructed with matrixes of Toeplitz type [10]. So, for finding the Shanks-Schmidt transformation $\sigma_{k, n}$ we have

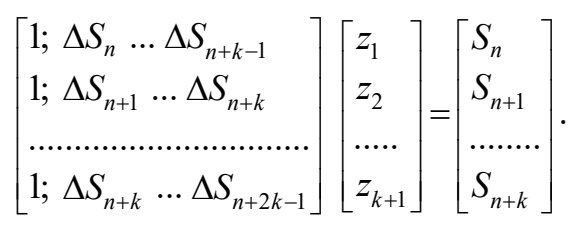

Once (15) has been solved, transformation follows the formula

$$
\sigma_{k, n}=1 /\left(z_{1}+z_{2}+\ldots+z_{k+1}\right) .
$$

The $T_{k, n}$ transformation is implemented using the following system 


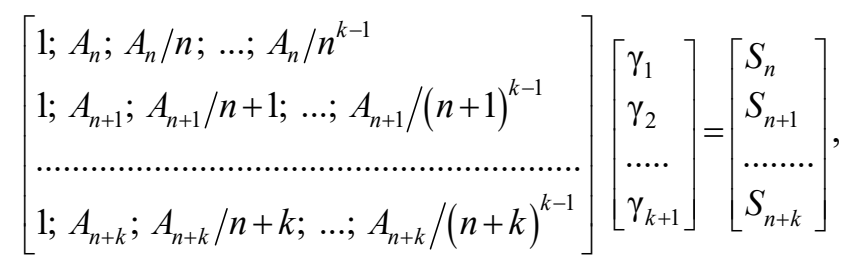

where $A_{n}=\Delta S_{n}, A_{n}=n \Delta S_{n}, A_{n}=\Delta S_{n} \Delta S_{n+1} / \Delta^{2} S_{n}$ for Levin's $t$-, $w$-, and $v$-transformations, respectively.

Actually, we can limit ourselves to finding only the first two components of solutions, $z_{1}$ and $\gamma_{1}$, as the rest $k$ components are required for analysis of the transformation spectrum.

\section{Transformation}

\section{of divergent sequences and series}

If solution of Eq. (1) has been obtained in the form of a series, the sequence $\gamma_{1(2 \mathrm{~m})}$ $(m=0,1,2, \ldots)$, as a rule, is divergent. Summation of such series and sequences is done using nonlinear transformations. Since representation $S(t)=\bar{S}+\sum_{k=1}^{v} \alpha_{k} \mathrm{e}^{\alpha_{k} t}$, where $\alpha_{k}$ is an arbitrary complex number, holds for $\left\{S_{n}\right\}$, then by analogy with the Fourier-series, the Fire summation is applied:

$$
\sigma_{n}=\frac{1}{n} \sum_{1}^{n} S_{k}
$$

For the series

$$
\sigma_{n}=\sum_{i=0}^{n} \frac{n-i+1}{n} a_{i} \varepsilon^{i}
$$

Sometimes we know the location of the singularity [1], giving rise to divergence of the series, on the axis or in the domain of variation of the parameter $\varepsilon$. A divergent series can be converted into an absolutely or conditionally convergent one by introducing some other comparison function. The Euler transformation [8] $\bar{\varepsilon}=\varepsilon /(1+\varepsilon)(\bar{\varepsilon} \in[0,0.5])$ applied to the series in (11) yields new series

$$
\bar{\psi}=\sum_{k=0}^{N}(-1)^{k} \Delta^{k} €_{0} \bar{\varepsilon}^{k} \cdot(1-\bar{\varepsilon}),
$$

where $\Delta a_{0}=a_{1}-a_{0} ; \quad \Delta^{2} a_{0}=a_{2}-2 a_{1}+a_{0} ; \ldots ; \quad \Delta^{k} a_{0}=\sum_{j=0}^{k} €_{k-j}(-1)^{j} c_{k}^{j} ; \Delta^{k}$ is the operator of finite differences of order $k$, and $C_{k}^{j}$ are the binomial coefficients. Reiterating the procedure $\tilde{\varepsilon}=\bar{\varepsilon} /(1+\bar{\varepsilon})$ it is feasible to eventually obtain a uniform approximation for the solution at the extremes of the specified interval $\bar{h}$. The source series is to be represented in the form $\psi=\sum_{k=0}^{N}(-1)^{k} a_{k} \varepsilon^{k}$.

After grouping the terms with the same power $\bar{\varepsilon}$ and dropping the terms of order $O\left(\bar{\varepsilon}^{N}\right)$ we finally obtain the following approximating expressions for the foil lift force and pitching momentum as an influence function of the $\bar{h}$ interval at small angles of attack: 


$$
\begin{aligned}
\bar{\psi}_{\gamma}= & 1+\bar{\varepsilon}+\frac{3}{2} \bar{\varepsilon}^{2}+\frac{11}{4} \bar{\varepsilon}^{3}+\frac{175}{32} \bar{\varepsilon}^{4}+\frac{177}{16} \bar{\varepsilon}^{5}+\frac{1423}{64} \bar{\varepsilon}^{6}+\frac{11323}{256} \bar{\varepsilon}^{7}+\frac{715923}{8192} \bar{\varepsilon}^{8}+ \\
& +\frac{7870829}{32768} \bar{\varepsilon}^{9}+\frac{56910769}{65536} \bar{\varepsilon}^{10}+\frac{530324997219}{524288} \bar{\varepsilon}^{11}+\frac{30926120625699}{2097152} \bar{\varepsilon}^{12}+ \\
& +\frac{1887973458979331}{16777216} \bar{\varepsilon}^{13}+\ldots ; \\
\bar{\Psi}_{M}= & 1+\frac{1}{2} \bar{\varepsilon}+\bar{\varepsilon}^{2}+\frac{31}{16} \bar{\varepsilon}^{3}+\frac{121}{32} \bar{\varepsilon}^{4}+\frac{239}{32} \bar{\varepsilon}^{5}+\frac{951}{64} \bar{\varepsilon}^{6}+\frac{60675}{2048} \bar{\varepsilon}^{7}+\frac{483543}{8192} \bar{\varepsilon}^{8}+ \\
& +\frac{2108939}{16384} \bar{\varepsilon}^{9}+\frac{20321375}{65536} \bar{\varepsilon}^{10}+\frac{128639927981}{524288} \bar{\varepsilon}^{11}+\frac{7737516436603}{2097152} \bar{\varepsilon}^{12}+ \\
& +\frac{240090786810183}{8388608} \bar{\varepsilon}^{13}+\ldots .
\end{aligned}
$$

While these series are slow to converge for large $\bar{h}$, they are uniformly valid in a larger domain. Therefore when nonlinear methods of convergence acceleration are employed even Aitken's transformation can yield the best approximation, with an accuracy of up to 23 significant figures for $\bar{h} \in[0.02,0.1]$. The accuracy improves for larger $\bar{h}$.

Let us now write an analytical expression for the sequence $\sigma_{1, n}(n=0,1,2, \ldots, N-1)$ derived from the series $\psi=\sum a_{m} \varepsilon^{m}$.

Constructing a sequence of partial sums and substituting it into $(-1)^{k} \Delta^{k} S_{n}>0$, where $\Delta^{k}=\sum_{j=0}^{k}(-1)^{j} C_{k}^{j}$ is the operator of central difference of order $k, C_{k}^{j}$ are the binominal coefficients,
we get

$$
\sigma_{1, n}=\sum_{0}^{n} b_{m} \varepsilon^{m} /\left(a_{n}-a_{n+1} \varepsilon\right),
$$

where $b_{0}=a_{0} a_{n} ; \quad b_{1}=a_{1} a_{n}-a_{0} a_{n+1} ; \ldots ; b_{m}=a_{m} a_{n}-a_{m-1} a_{n+1}$.

If in (21) $a_{n}-a_{n+1} \varepsilon \equiv 0$, then the nominator of the fraction should be examined for zeroes. Suppose, there is $\varepsilon_{\text {кр }}=a_{n} / a_{n+1}$ among the polynomial zeroes; this defect can be readily cured. When $\varepsilon_{\text {кр }}$ is a pole, we should set $\sigma_{1, n}=\sigma_{1, n-1}$. Sometimes appearance of a pole indicates the limits of series convergence. Thus for the $\psi_{Y}$ series from (11) these are $\varepsilon_{\mathrm{\kappa p}}=a_{n} / a_{n+1}$, which agrees with the results obtained by other techniques $[3,4]$.

The amount of information that can be derived for a given number of approximations does not appear to be enough for a transformation to ensure the best approximation over the entire domain of variation of parameter $\varepsilon$. We then have to approach the true solution via various transformations for each $h$ value.

The Shanks-Schmidt-Levin transformations are based on rational approximation of the series $\sum a_{k} x^{k}$. They complement each other as their representation error is associated with the Loran-series expansion of the function

$$
f(z)=\sum_{k=0}^{\infty} a_{k} z^{k}+\sum_{k=1}^{\infty} b_{k} z^{-k}
$$

The first term in (22) refers to errors under Shanks and second one under Levin transformations. In a general case, it is necessary to have available these transformations along with the recursive techniques for their evaluation to be able to automatically monitor the situation. If it appears that the 
Shanks transformation fails or is slow to converge for $n_{i} \rightarrow \infty$, i.e. $\left(\sigma_{k, n_{i}}-\sigma_{k, n_{i+1}}\right)>\varepsilon$, we should then switch to the Levin transformation.

If Fire summation (19) is applied to series (11) and (20) followed by summation for each $\bar{h}$ using Shanks-Schmidt and Levin's algorithms then the result we obtain will agree with that of numerical simulation. The best analytic approximations employing transformation of $\sigma_{k, n}$ series (20) are obtained with rational fractions $\sigma_{1,3} ; \sigma_{1,6} ; \sigma_{2,7} ; \sigma_{3,5} ; \sigma_{3,8}$. The figure shows the influence function $\psi_{Y}$ as evaluated by (11). Also shown are the results of Fire summation applied to series (20) followed by summation using the Shanks-Schmidt-Levin algorithm. Computational results based on this algorithm are in very good agreement with the numerical result for integral equation (1) solved by the collocation technique using BCF apparatus as prescribed by the given algorithm. The four-term expansion $\psi_{Y}$ from (11) is uniformly valid over the interval $\bar{h} \in\left(\bar{h}_{k p}^{(1)}, \bar{h}_{k p}^{(2)}\right)$, the quantitative result, however, being far from exact.

\section{Conclusion}

Optimal asymptotics contains nine terms, and still it rapidly deviates from the exact solution; however an approximation such as $\sigma_{3,8}$ for $h<0,01$ already gives a relative error less than $1 \%$.

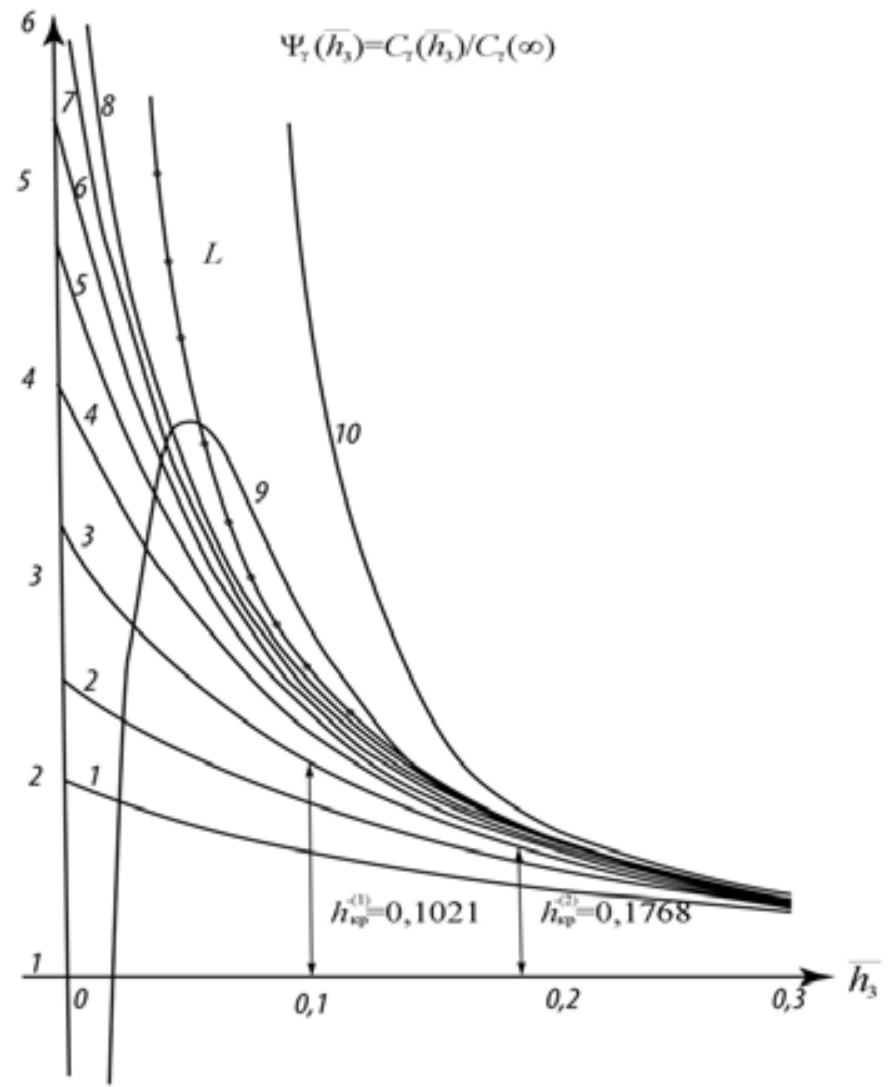

Fig. The function of influence of $\bar{h}$ interval on the lift force: $\underline{L}$ - influence function derived from (20) using Levin's transformation for each $\bar{h}$ - Numerical simulation results for Eq. (1) obtained by the collocation method using the BCF apparatus 
The discussed algorithms have been implemented as computational programs for algebraic (ALFA) and integral (OMEGA) equations, ordinary (SIMP) and improper (SECOB) integrals, including the Cauchy integral (DSECOB) as well as summation programs for sequences and series (SHENKS, AYTKEN), including divergent ones (EULER).

The reported study was funded by Russian Foundation for Basic Research, Government of Krasnoyarsk Territory, Krasnoyarsk Region Science and Technology Support Fund to the research projects № № 17-48-240386 p_a and 16-41-242156 p_opu_M.

\section{References}

[1] Van Dyke M. Perturbation Methods in Fluid Mechanics. M.: Mir, 1967. 310 p. (in Russian).

[2] Demidenko N.D., Kulagin V.A., Shokin Y.I., Li F.-C.Heatandmass transfer and supercavitation. Novosibirsk: Nauka, 2015. 436 p. (in Russian).

[3] Kulagin V.A., Vil'chenko A.P., Kulagin T.A. Modeling of two-phase supercavitation flows; Editor V.I. Bykov. Krasnoyarsk: KSTU, 2001. 187 p. (in Russian).

[4] Ivchenko, V.M., 1985, Supercavitation mechanism hydrodynamics, Irkutsk, Irkutsk Univ. Publishing Comp. 232 p. (in Russian).

[5] Ivchenko V.M., Kulagin V.A., Nemchin A.F. The cavitational technology; Ed. Acad. G.V. Logvinovich. Krasnoyarsk: KSU Publishing House, 1990. 200 p. (in Russian).

[6] Shanks D. Non-linear transformation of divergent and slowly convergent equences, J. Math. and Phys., 1955, fasc. 34. 1-42.

[7] Ogirko O.V. Application and refinement of nonlinear cubature formulae, Institute of applied problems in mechanics and mathematics AS Ukraine. Lviv, 1982. 18 c. Dep. with VINITI No 324-82 (in Russian).

[8] Krylov V.I. et al. Computational Methods. V. II. M.: Nauka, 1977. 399 p. (in Russian).

[9] Skorobogatko V.Ya. The theory of branched continued fractions and its application to computational mathematics. M.: Nauka, 1983. 311 p. (in Russian).

[10] Hunter C., Guerrieri B. Deducing the properties of singularities of function from their Taylor series coefficients, J of Applied Math., 1980, v. 39, No 2. 248-263.

[11] Verlan A.F., Sizikov V.S. Methods for solving integral equations with machine computational software. Kiev: Naukova dumka, 1978. 292 p. (in Russian).

[12] Frank de Hoog. A new algorithm for solving Toeplitz systems of equations, Linear Algebra and its Applications, April, 1987. Vol. 88-89, 123138: doi.org/10.1016/0024-3795(87)90107-8. 